\title{
Grabbing attention without knowing: Automatic capture of attention by subliminal spatial cues
}

Mulckhuyse, M., Talsma, D., \& Theeuwes, J. (2007). Grabbing attention without knowing: Automatic capture of attention by subliminal spatial cues. Visual Cognition, 15(7), 779-788. 


\begin{abstract}
The present study shows that an abrupt onset cue that is not consciously perceived can cause attentional facilitation followed by inhibition at the cued location. The observation of this classic biphasic effect of facilitation followed by inhibition of return (IOR) suggests that the subliminal cue captured attention in a purely exogenous way. Since IOR is not observed following endogenous shifts of spatial attention, but is observed following exogenous, stimulus-driven shifts of spatial attention, it is unlikely that top-down control settings or other non-attentional effects played a role. The current findings are interpreted in terms of a neurobiological model of visual awareness.
\end{abstract}


Introduction

It is well-known that abrupt onsets can capture attention in an exogenous way (see e.g., Theeuwes, 1991, 1994b; Yantis \& Jonides, 1990; but see Folk, Remington, \& Johnston, 1992). Peripheral cueing paradigms demonstrate that when a visual abrupt onset is used as a cue, spatial attention exogenously shifts to the cued location (e.g., Posner, 1980). In this paradigm, participants fixate the centre of the screen while a salient abrupt onset cue appears briefly on either the left or the right of fixation. After a short delay a target is presented. Even though the cue does not contain any information about the upcoming location of the target, participants are faster and more accurate in responding to targets that appear at the cued than at the uncued location, sometimes labelled as the facilitation effect. Furthermore, if there is a delay between the offset of the cue and the onset of the target, participants are slower and less accurate in responding to targets at the cued than at the uncued location, called inhibition of return (IOR; cf. Posner \& Cohen, 1984). It is important to note that IOR at a location in space only follows after attention has shifted reflexively to that location (see Klein, 2000, for a review). Indeed, IOR does not follow a shift of attention that is directed endogenously (voluntarily; Posner \& Cohen, 1984; Pratt, Kingstone, \& Khoe, 1997), except in conditions in which participants endogenously prepare an eye movement (Rafal, Calabresi, Brennan, \& Sciolto, 1989).

Recently, spatial cueing paradigms were used with peripheral cues that were not consciously perceived. Although these cues did not reach subjective awareness, the results became part of a discussion as to whether visual onsets capture attention automatically, or capture attention only when they are contingent on top-down goals. For example, McCormick (1997) investigated the attentional effects of cues above and below subjective threshold. In order to separate endogenous from exogenous orienting of attention, he used an informative cue. When participants were unaware of the peripheral cues, a facilitation effect at unexpected locations was found, but there was no IOR. He reasoned that the absence of IOR may be caused by top-down strategy used by the participants. However, the facilitation effect was induced by exogenous attentional capture of the cue. A potential problem of McCormick's study was the fact that part of the participants task was to report whether or not they had detected the cue after each trial. Therefore, the cue may have captured attention because it was part of the attentional set.

Ivanoff and Klein (2003) specifically addressed this issue using a spatial cueing paradigm. Participants first completed a condition in which they performed a go/no go task without reporting the presence or absence of the masked peripheral cue. Subsequently, they performed the same task in which they were also required to report whether or not they had detected the cue. Ivanoff and Klein found a facilitating effect, but no IOR, when cue report 
was part of the task and found IOR, but no facilitation effect, when cue report was not part of the task. In their view, the cue caused an exogenous shift of attention in both conditions, but attention was disengaged rapidly when the cue was task irrelevant. They argued that the facilitation effect could not be found because it was combined with early IOR (as in Danziger \& Kingstone, 1999). In contrast, attention would remain engaged at the cued location when the cue was task relevant and thereby causing the facilitation effect, but no IOR. Based upon these findings, they concluded that a facilitation effect can only be found when the cue is part of the attentional set (Folk et al., 1992).

More recently, Ansorge and Neumann (2005) tried to distinguish between facilitation effects due to top-down goals and facilitation effects due to bottom-up or stimulus driven capture. They used a spatial cueing paradigm with metacontrasted primes in which the target was the mask and the primes were smaller replicas of the target. In line with the direct parameter specification theory (DPS; Neumann, 1990), Ansorge and Neumann reasoned that the prime could give information about the appropriate response, or the prime could be contingent on top-down goals. Therefore, they systematically reduced the information the prime could give. Assessment of awareness of the primes was performed after the reaction time task. First, they found that the facilitation effect of cue validity (same location as the target) disappeared when the prime differed from the target in colour - although they did find a nonsignificant difference in the direction predicted by the bottom-up capture view. Second, the facilitation effect of validity also disappeared when the position of the prime gave no information about the appropriate response. Third, they found a significant facilitation effect only when the position of the prime gave information about the appropriate response. Ansorge and Neumann concluded that attention was not captured exogenously by these primes, but was captured only when it fits the topdown attentional setting.

In the current study, we wanted to determine whether it is possible to obtain cueing effects in a task in which the nonconsciously perceived cue captures attention in a bottom-up fashion. Therefore we designed a paradigm in which the cue did not resemble the target, gave no information about the appropriate response, was uninformative about the location of the upcoming target, and in which cue report was not part of the task. In addition, we employed a novel approach to present cue stimuli subliminally. One of the three discs that served as placeholders was presented just an instant earlier. We expected this disc to attract attention by its sudden onset and thereby serve as a cue. Because the other two discs followed immediately, it gave the impression that all three discs appeared simultaneously. For this reason, we expected that the "cue" would not be perceived consciously. If exogenous nonconscious attentional cueing effects exist, we expected to find faster detection when the target appears immediately after the cue at the cued location than when it appears at the uncued location. Furthermore, if this shift of attention is indeed exogenous, 
with a long delay we expected the occurrence of IOR, i.e., slower detection of the target at the cued location than at the uncued location.

Method

\section{Participants}

Sixteen paid volunteers (aged 18_24) participated in the experiment. All participants had normal or corrected to normal vision.

\section{Apparatus and design}

The stimuli were presented on a 19-inch monitor using a PC with a 1024_ 768 resolution and a refresh rate of $120 \mathrm{~Hz}$. E-Prime software (Psychology Software Tools) was used for stimulus presentation and data recording. All stimuli were presented on a grey background (x_0.286, y_0.322: Luminance $4.6 \mathrm{~cd} / \mathrm{m} 2$ ). During the experiment, two different tasks were administered, first a subliminal cueing task followed by a "cue report" task. The subliminal cueing task consisted of five blocks of 40 trials. Each condition consisted of 40 trials which were randomly presented during the experiment. The cue was not informative of the location of the upcoming target. Catch trials (20\%) were included to avoid anticipation. Each trial began with a black fixation cross in the centre of the screen that stayed on for $1000 \mathrm{~ms}$ (see Figure 1). The fixation cross disappeared for $200 \mathrm{~ms}$, after which one of the discs was presented. The disc consisted of a grey filled circle, 1.98 in diameter (x_0.287, y_0.315: Luminance $12.7 \mathrm{~cd} / \mathrm{m} 2$ ) that was presented for $16 \mathrm{~ms}$ either 6.78 to the left or to the right of the centre of the screen. Following this first disc, a display consisting of two more discs, each with the same size and luminance as the first disc, was presented. The resulting display containing the three discs was positioned in a straight line with their centres separated by 6.78. Either simultaneously with the onset of the latter two discs, or after an SOA of $1000 \mathrm{ms,}$ a target stimulus appeared.

This target consisted of a small black dot that could appear inside either the left or right disc. After $80 \mathrm{~ms}$, the target stimulus was extinguished and after another $200 \mathrm{~ms}$ the discs disappeared and the grey background was presented for 1000 ms before the next trial began. The "cue report" task consisted of four blocks of 20 trials that were identical to the detection time task, including the $20 \%$ catch trials without a target, with the exception that the trial ended when a response was given.

\section{Procedure}

Participants were seated $75 \mathrm{~cm}$ from the computer screen with their head positioned on a chinrest. They were explicitly instructed to remain fixated on the centre of the screen. Participants had to press the space bar as soon as they detected the target. Note that they 
were not informed about the temporal difference in onset of one of the discs. The experiment started with a practice block of six trials. If participants pressed the spacebar before the presentation of the target, or if they responded to late (i.e., more than $630 \mathrm{~ms}$ after target onset), a sound was presented indicating a wrong response.

After conducting the subliminal cueing task, we assessed whether participants were able to perceive the earlier onset of one of the discs when they were instructed to do so. Participants were asked to ignore the target, but to indicate which of the discs, the left or the right, was presented an instant earlier than the other two discs, by pressing respectively the " $z$ " key or the " $m$ " key. In half of the trials the disc on the left was presented an instant earlier and in the other half of the trials on the right. Each trial ended when a response was given and no sound was presented when given a wrong response.

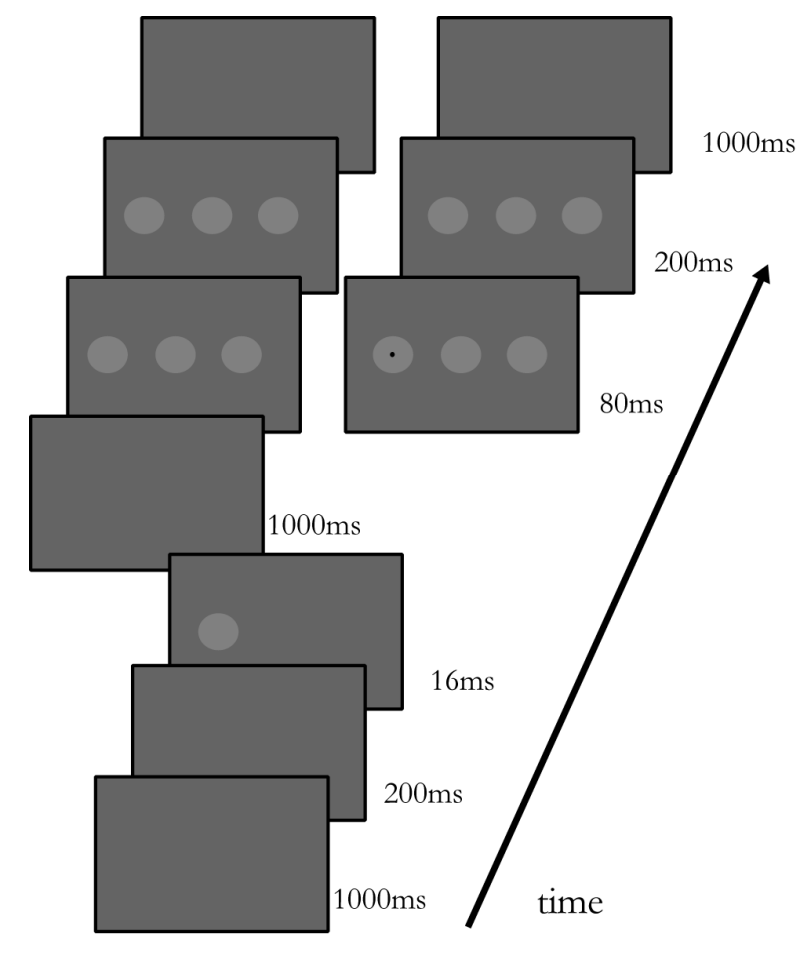

Figure 1. From bottom to top, succession of events in a trial in which the cue happened to be valid. On the left the sequence in a trial with a long SOA is depicted, on the right a trial with a short SOA.

Results

\section{Cue report task}

Participants all gave a subjective report afterwards about not being able to perceive the cue. A one-tailed binomial test for each participants revealed that none of the participants scored 
significantly above chance level. Mean detection performance in the cue report task was $50 \%$ (min. 44\% and max. 59\%) and not significantly above chance level ( $p=.98)$.

\section{Subliminal cueing task}

Response reaction times with a latency of less than $100 \mathrm{~ms}$ and a latency of more than 630 $\mathrm{ms}(0.4 \%$ of all trials) were omitted from analysis. Mean response error rate on the catch trials was $3.4 \%$. A repeated-measures analysis of variance (ANOVA) with SOA (short and long) and cue validity (cued location vs. uncued location) on detection time showed a significant main effect of $S O A, F(1,15)=69.5, p<.01, M S E=816.8$, and a significant interaction effect of SOA and cue validity, $F(1,15)=20.9, p<.01, \mathrm{MSE}=46$ (see Figure 2).

Planned comparisons showed a facilitation effect at the short SOA; detection times at the cued location were faster than at the uncued location (402 ms vs. $413 \mathrm{~ms}$ ), $\mathrm{t}(15)=2.79$, $p<.05$. More importantly, at the long SOA this effect reversed: At the cued location detection times were slower than at the uncued location (350 ms vs. $345 \mathrm{~ms}$ ), t (15)=2.24, $\mathrm{p}<.05$.

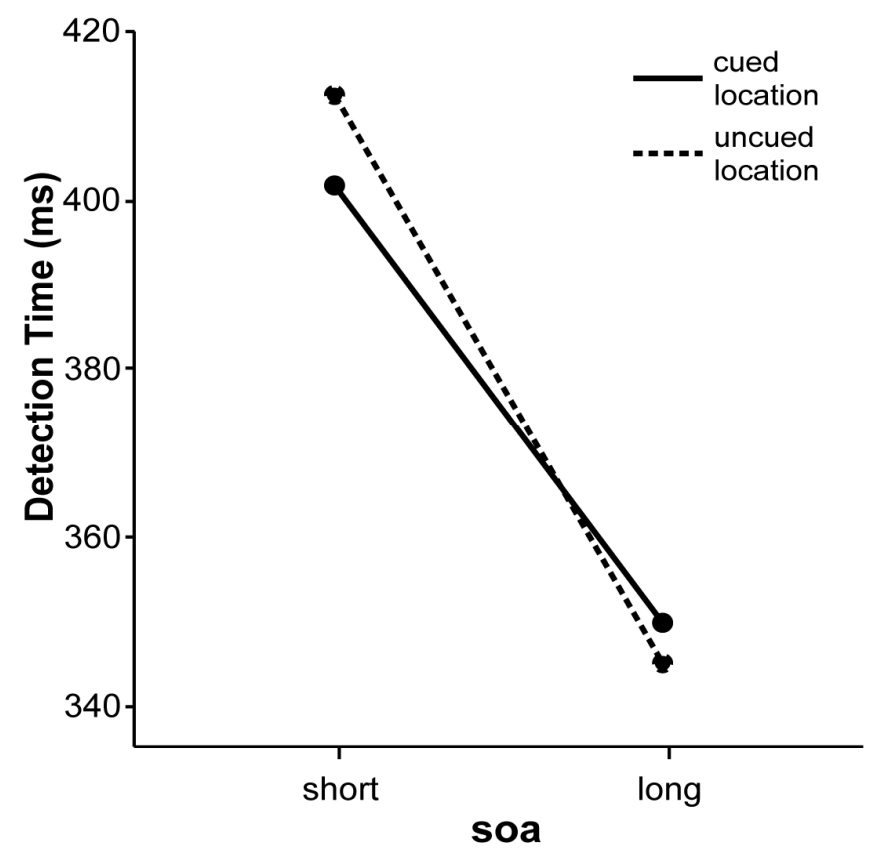

Figure 2. Mean response detection time at the cued location (solid line) and the uncued ocation (dotted line) with a short and long SOA. 
The present study shows that cueing effects typically obtained in peripheral cueing paradigms (e.g., Posner, 1980) persevere even when the peripheral cue is not consciously perceived. Although the effects are not as pronounced as when a visible cue is used, the classic biphasic effect of facilitation followed by inhibition is clearly observed. Whereas previous studies using subliminal cues reported ambiguous results with respect to the occurrence of facilitation and inhibition (e.g., Ivanoff \& Klein, 2003), the current study is the first to show the classic effect of facilitation followed by inhibition using subliminal cues. Since it is generally agreed that IOR is the product of reflexive, involuntary orienting, the current findings also provide evidence that subliminal cues can cause exogenous attentional orienting.

One could argue that the cue we used was part of the attentional set because (1) the cue was presented at a possible target location and (2) the cue and the target were both defined by onsets. Even though we cannot completely rule out the possibility that attentional set played a role, it should be noted that the cue was uninformative and we did find IOR. As noted, IOR is associated with reflexive shifts of spatial attention (Theeuwes \& Godijn, 2002).

There may be several reasons why previous studies did not find a facilitation effect (Ansorge \& Neumann, 2005; Ivanoff \& Klein, 2003). Contrary to earlier studies, we used a relatively short SOA (i.e., $16 \mathrm{~ms}$ ) and presented the target immediately following the cue instead of using an interval between cue and target. This corresponds with Ivanoff and Klein's conclusion that attention at the cued location is disengaged rapidly when the cue is not task relevant. Theeuwes, Atchley, and Kramer (2000) investigated the time course of disengagement of a (visible) distractor that captured attention in a visual search task by using different SOAs between distractor and target and by manipulating the congruence between them. They found that although an attentional-set could not prevent attentional capture by a salient stimulus, it did assert its influence on the time course of disengagement of attention. The disengagement of attention from the distractor location took much longer when the distractor and target had the same defining properties. It is possible that this same process takes place in a short spatial cueing paradigm with subliminal cues. If the cues are not task relevant, a facilitation effect would only manifest itself if the cue is immediately followed by the target.

The reason why participants were unable to consciously perceive the cue, could be explained within the neurobiological model of visual attention and awareness by Lamme (2003). Presenting the disc for $16 \mathrm{~ms}$ without follow up of the other discs, is enough to lead to visual awareness. However, if the disc is followed immediately by the other two discs, there is no conscious perception of the first disc. Central to Lamme's model is the distinction between an initial feedforward sweep followed by recurrent processing, which is necessary 
for a visual stimulus to reach consciousness (Lamme \& Roelfsema, 2000). The visual information of the first disc could have been replaced by new visual information of the three discs before recurrent processing of the first disc had led to visual awareness (Di Lollo, Enns, \& Rensink, 2000). The exogenous shift of attention, caused by the cue, could also be explained within the model. The initial feedforward sweep can give rise to a reflex-like, unconscious output or modification of behaviour of information. The superior colliculus is one of the earliest activated areas by the feedforward sweep and is, among other brain structures, involved in attentional processes such as attentional capture, IOR, and oculomotor programming (see for reviews Klein, 2000; Shipp, 2004; van der Stigchel, Meeter, \& Theeuwes, 2006). It receives visual input directly from the retina as well as from the visual cortex and processes visual information via the quick pathway to the parietal cortex (Lamme \& Roelfsema, 2000). Given this line of reasoning, the initial feedforward activation induced by the first disc may have reached the superior colliculus, which produced the attentional effects. In fact, a study by Kentridge, Heywood, and Weiskrantz (1999) with a blindsight patient seems to imply that the superior colliculus is responsible for mediating these nonconscious attentional processes. The patient in this study responded faster to targets that appeared at a cued than at an uncued location, although he was neither aware of the cue nor of the target. Previously, this same patient was scanned in an fMRI study by Sahraie et al. (1997). They tested brain activity in his blind hemifield generated by visual events of which the patient was aware - meaning some sort of "feeling" that something has happened - and visual events of which the patient was unaware. They found that subcortical structures and in particular the superior colliculus were activated in trials in which the patient reported no awareness of a visual event. Because the damage to his visual cortex had been more than 30 years ago, one could argue that pathways mediating visual attention in the brain had changed. Nevertheless, future research with healthy participants using subliminal cueing stimuli could give more insight in the role of the superior colliculus and orienting attention without awareness. 


\section{References}

Ansorge, U., \& Neumann, O. (2005). Intentions determine the effect of invisible metacontrast-masked primes: Evidence for top-down contingencies in a peripheral cueing task. Journal of Experimental Psychology: Human Perception and Performance, 31, 762-777.

Danziger, A., \& Kingstone, A. (1999). Unmasking the inhibition of return phenomenon. Perception \& Psychophysics, 61 (6), 1024-1037.

Di Lollo, V., Enns, J. T., \& Rensink, R. A. (2000). Competition for consciousness among visual events: The psychophysics of reentrant visual processes. Journal of Experimental Psychology: General, 129 (4), 481-507.

Folk, C. L., Remington, R. W., \& Johnston, J. C. (1992). Involuntary covert orienting is contingent on attentional control settings. Journal of Experimental Psychology: Human Perception and Performance, 18, 1030-1044.

Ivanoff, J., \& Klein, R., M. (2003). Orienting of attention without awareness is affected by measurement-induced attentional control settings. Journal of Vision, 3, 32-40.

Kentridge, R. W., Heywood, C. A., \& Weiskrantz, L. (1999). Attention without awareness in blindsight. Proceedings of the Royal Society of London, Series B, Biological Sciences, 266, 1805-1811.

Klein, R. M. (2000). Inhibition of return. Trends in Cognitive Sciences, 4 (4), 138-147.

Lamme, V. A. F., \& Roelfsema, P. R. (2000). The distinct modes of vision offered by feedforward and recurrent processing. Trends in Neurosciences, 23 (11), 571-579.

Lamme, V. A. F., (2003). Why visual attention and awareness are different. Trends in Cognitive Sciences, 7 (1), 12-18.

McCormick, P. A. (1997). Orienting attention without awareness. Journal of Experimental Psychology: Human Perception and Performance, 23, 168-180.

Neumann, O. (1990). Direct parameter specification and the concept of perception. Psychological Research, 52, 207-215.

Posner, M. I. (1980). Orienting of attention. Quarterly Journal of Experimental Psychology, 32, 3-25.

Posner, M. I., \& Cohen, Y. (1984). Components of visual orienting. In H. Bouma \& D. Bowhuis (Eds.), Attention and Performance X (pp. 531-556). Hillsdale, NJ: Erlbaum.

Posner, M. I., Rafal, R. D., Choate, L. S., \& Vaughan, J. (1985). Inhibition of return: neural basis and function. Cognitive Neurophysiology, 2 (3), 211-228.

Pratt, J., Kingstone, A., \& Khoe, W. (1997). Inhibition of return in location- and identitybased choice decision tasks. Perception \& Psychophysics, 59 (6), 964-971.

Rafal, R. D., Calabresi, P. A., Brennan, C. W., Scioloto, T. A. (1989). Saccade preparation inhibits reorienting to recently attended locations. Journal of Experimental Psychology: Human Perception and Performance, 15 (4), 673-685.

Sahraie, A., Weiskrantz, L., Barbur, J. L., Simmons, A., Williams, S. C. R., \& Brammer, M. J. (1997). Pattern of neural activity associated with conscious and unconscious processing of visual signals. Proceedings of the National Academy of Sciences, 94, 9406-9411.

Shipp. S. (2004). The brain circuitry of attention. Trends in Cognitive Sciences, 8 (5), 223230.

Theeuwes, J. (1991). Exogenous and endogenous control of attention: The effect of visual onsets and offsets. Perception \& Psychophysics, 49 (1), 83-90.

Theeuwes, J. (1994). Stimulus-driven capture and attentional set: Selective search for color and visual abrupt onsets. Journal of Experimental Psychology: Human Perception and Performance, 20, 799-806.

Theeuwes, J., Atchley, P. \& Kramer, A.F. (2000). On the time course of top-down and bottom-up control of visual attention. In S. Monsell \& J. Driver (Eds.), Attention \& Performance, 18 (pp. 105-125) Cambridge, MA: MIT Press.

Theeuwes, J., \& Godijn, R. (2002). Irrelevant singletons capture attention: Evidence from inhibition of return. Perception \& Psychophysics, 64 (5), 764-770.

Van der Stigchel, S., Meeter, M., Theeuwes, J. (2006). Eye movement trajectories and what 
they tell us. Neuroscience and Biobehavioral Reviews 30, 666-679.

Yantis, S., \& Jonides, J. (1990). Abrupt visual onsets and selective attention: voluntary versus automatic allocation. Journal of Experimental Psychology: Human Perception and Performance16 (1), 121-134. 
Acknowledgements

This research was funded by a grant from Human Frontier Science Program (HSFP-

RGP39/2005) to Jan Theeuwes. 